\title{
Hepatitis C virus epidemiology in Yemen: Systematic review
}

\author{
Yemen'de hepatit C virüsü epidemiyolojisi: Sistematik derleme
}

\author{
(D) Amtalsaboor A. A. ALMAHBASHI \\ Ankara University, Biotechnology Institute, Ankara
}

\begin{abstract}
Bacground and Aims: To identify and well-characterize the prevalence of hepatitis $C$ virus infection in different populations in Yemen. Also, to determine the future epidemiological study areas in Yemen. Materials and Methods: A systematic review was conducted with the use of the PRISMA guidelines. Between 1980 to 2020, a comprehensive search was done for any epidemiological data on hepatitis $C$ in Yemen that were conducted in each PubMed, web of Science, Scopus, and Google Scholar. Results: Forty-nine (49) studies were matching the inclusion and exclusion criteria from the four databases. After analyzing these 49 studies we found that in the general group of the population the prevalence was ranged between $0.07 \%-8.5 \%$. Where the highest prevalence was in pregnant women (up to $8.5 \%$ ) then the public (up to $6 \%$ ) then blood donors and children up to $3 \%$ and $2.8 \%$ respectively. In the intermediate-risk group, hepatitis C virus infection prevalence was ranged between $1.03 \%-3.5 \%$ where all population was from the health department workers. In the high-risk group, the range was between 6.4\%-62.7\%, and the highest prevalence was recorded in liver disease patients. In the patient's group the range of hepatitis $C$ virus infection prevalence was between $0 \%-46 \%$, while the sickle cell anemia patients were the lowest prevalence the chronic renal failure patients had the highest prevalence. Conclusion: This systemic review demonstrates a high prevalence of hepatitis C infection in Yemen. There is also a need to expand the scope of research and pay attention to new research groups and other cities to get a more comprehensive idea of the prevalence of the hepatitis C virus in Yemen.
\end{abstract}

Key words: Hepatitis C virus, epidemiology, Yemen, systematic review

\section{INTRODUCTION}

Hepatitis C virus (HCV) is causing chronic liver disease, hepatocellular carcinoma, and cirrhosis. where this virus is a blood-borne disease and progresses from acute to chronic infection in the liver. Also, it associates with several morbidities, and it is the reason for a lot of liver transplantation in many countries $(1,2)$. Also, it considers as The seventh leading cause of death globally and the fifth in the Middle East and North Africa (MENA) $(3,4)$. Globally about 71 million people infected with HCV where the most affected region is MENA region with nearly 15 million infected persons (5). Even though the related mor-

Correspondence: Amtalsaboor A. A. ALMAHBASHI

Ankara University, Biotechnology Institute,

Gümüşdere 60. YII Yerleșkesi, Keçiören/ ANKARA

E-mail: amtalsaboor1@gmail.com
Giriş ve Amaç: Yemen'deki farklı popülasyonlarda hepatit C virüsü enfeksiyonunun prevalansını belirlemek, iyi karakterize etmek ve ayrıca, Yemen'de gelecekteki epidemiyolojik çalışma alanlarını belirlemektir. Gereç ve Yöntem: PRISMA yönergelerinin kullanımıyla sistematik bir inceleme yapıldı. Yemen'deki hepatit C ile ilgili; PubMed, web of Science, Scopus ve Google Scholar'da 1980 ile 2020 arasında yürütülen, herhangi bir epidemiyolojik veri için kapsamlı bir araştırma yapıldı. Bulgular: Kırk dokuz (49) çalışma, dört veri tabanındaki dahil etme ve dışlama kriterleriyle eşleşti. Bu 49 çalışmanın analizi ile nüfusun genel grubunda prevalansın \%0.07-\%8.5 arasında değiştiğini bulduk. En yüksek prevalansın hamile kadınlarda olduğu (\%8.5'e kadar), ardından genel (\%6'ya kadar), ardından da sırasıyla \%3 ve \%2.8 ile kan bağışçıları ve çocuklarda olduğu görüldü. Orta risk grubunda, tüm popülasyon sağlık departmanı çalışanlarından olmak üzere, hepatit C virüsü enfeksiyonu prevalansı \%1.03-3.5 arasında değişiyordu. Yüksek riskli grupta, prevalans aralığı \%6.4-\%62.7 arasında olup, en yüksek prevalans karaciğer hastalığı olanlarda kaydedilmiştir. Hasta grubunda hepatit C virüsü enfeksiyonu prevalansı aralığı \%0-\%46 arasındayken, orak hücreli anemili hastalar en düşük prevalansa, kronik böbrek yetmezliği olan hastalarsa en yüksek prevalansa sahipti. Sonuç: Bu sistemik derleme, Yemen'deki hepatit $C$ enfeksiyonu prevalansının yüksek olduğunu göstermektedir. Yemen'deki hepatit C virüsünün prevalansı hakkında daha kapsamlı bir fikir edinmek için araştırma kapsamını genişletmeye ve yeni araştırma gruplarına ve diğer şehirlere dikkat etmeye ihtiyaç vardır.

Anahtar kelimeler: Hepatit C virüs, epidemiyoloji, Yemen, sistematik derleme

tality of HCV strains healthcare systems worldwide, the Direct-acting antivirals (DAAs) give promising prospects to clear HCV infection and reduce HCV disease burden in the soon future (6). Whereas the elimination of hepatitis C virus by 2030 is a global goal for the World Health Organization (WHO). The epidemiology of hepatitis $\mathrm{C}$ in Yemen is still not well described, and it needs to define the prevalence in different populations and define the areas of a future epidemiological study in Yemen which are the objectives of this systematic review $(7,8)$.

ALMAHBASHI AAA. Hepatitis C virus epidemiology in Yemen: Systematic review. The Turkish Journal of Academic Gastroenterology 2021;20:49-58. DOI: 10.17941/agd.923259

Manuscript received: 20.04 .2021 - Accepted: 27.04 .202 


\section{MATERIALS and METHODS}

The preparation of this systematic review was done according to the PRISMA guideline (Preferred Reporting Items for Systematic Reviews and Meta-Analyses) (9). The articles were searched in four main biomedical and health databases of Scopus, PubMed, Web of Science, and Google Scholar. In the first three databases, the research was done without any language restriction, but the period of the search was restricted from 1980 to the end of 2020 because the discovery of HCV was in 1989 (10). The search was done using the terms of "hepatitis $C$ or HCV or anti-HCV or HCV antibody or core HCV antigen or HCV CAg or HCV RNA and Yemen or limited to Yemen" in the three databases to determine all publications about HCV from Yemen. All identified articles from these databases were exported to endnote for processing. But in Google Scholar, the research was done by using keywords of "hepatitis C or HCV and Yemen" and the same period restriction as in the other database by screening the titles of all the articles that could be related to our inclusion and exclusion criteria and then exported to the Endnote. The duplicate articles were identified and excluded from all the articles in the Endnote. The remaining unique articles were undergone to a two-stage screening process. In the first stage, all the articles' titles and abstracts were screened for relation to the aim. The related or it could be related articles proceeded to the next stage of screening, wherein this stage full-texts were evaluated according to our inclusion/exclusion criteria. Eligible articles were included in this study, and ineligible articles were excluded according to criteria. The summary of the whole process can be found in the diagram in Figure 1 that was produced according to the PRISMA guideline.

\section{The Inclusion and Exclusion Criteria}

Briefly, every article reporting any epidemiological data of the prevalence and incidence of Hepatitis $C$ virus in Yemen was included. The excluded articles were case reports, case series, reviews, articles that contained duplicate information, articles that their full text is not available, and if the study population was Yemeni nationals residing outside Yemen.

\section{Data Analysis and Population Classification}

The rest articles were analyzed by identifying and presenting as tables in Excel included these data: authors, year of sample collection, year of publishing, type of study, sample size, prevalence, study site, prevalence by gender, Governorate, population, and test type. Then the population was divided into four groups 1-General population (low-risk group): included blood donors, public people, pregnant women, children, and healthy controls. 2-Inter-

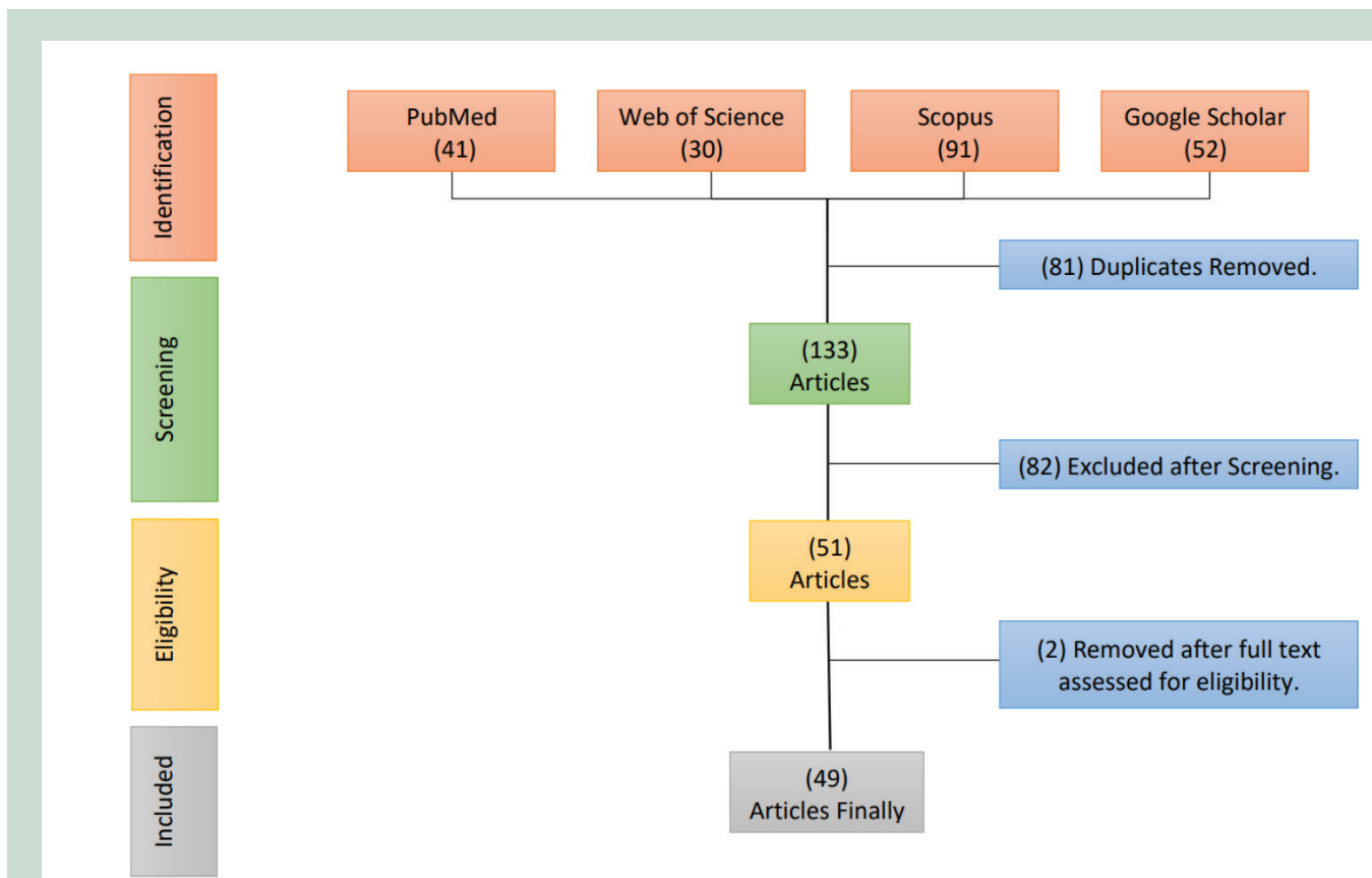

Figure 1. PRISMA's diagram for HCV epidemiology in Yemen. 
mediate risk group: included workers in various medical fields or in health departments. 3- High-risk group: included patients with hepatocellular carcinoma, liver disease, and hemodialysis. 4- Patient from other diseases: included all other patients from different clinics and diseases.

\section{RESULTS}

The results and the steps for the articles search were summarizing in flow diagram (Figure 1). From the four databases a total of 214 articles were identified; about 81 duplicates articles were removed, then 82 articles were removed after the title and abstract screening. The remaining 51 publications that met the inclusion criteria were screened again with the full text, two of them were excluded due to not meeting the inclusion criteria. Finally, 49 studies were meeting the inclusion criteria for result analysis.

From our result we can found that in the year of publishing the period between 2016-2020 was having the highest publishing numbers $(n=20,40.8 \%)$ then 2011 2015 with $(n=16,32.6 \%)$, where 2006-2010, 2001 2005, 1996-2000, and 1990-1995 have the number of publishing $n=5, n=3, n=2$, and $n=3$ respectively (Figure 2). While the study type of all the researches were cross-sectional studies except one research was case-control where it has a $9.9 \% \mathrm{HCV}$ of prevalence in it (11). Also, among the studies reporting the prevalence of hepatitis $C$ by gender ( $n=30$ studies) the prevalence range for both genders was similar, where it was in the females between $0 \%-62.5 \%$ and the males between $0 \%$ $63.3 \%$. According to the governorate, about 27 studies were conducted in the Sana'a governorate altogether involved a prevalence range between $0.2 \%-54.2 \%$. The governorate distribution of studies was Aden $(n=8)$, Alhudaydah $(n=6)$, Ibb $(n=5)$, Taiz $(n=4)$, Hadhramaut $(n=2)$, Hajjah $(n=2)$, and for each of Amran, Dhamar, Raymah, Sa'dah, and Socotra one study as seen in (Figure 3). Where sample sizes across the forty-nine stud-

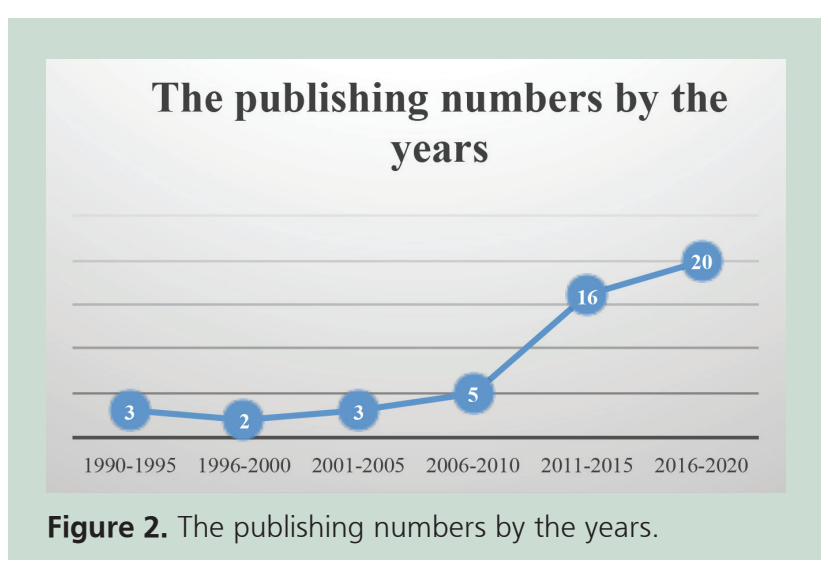

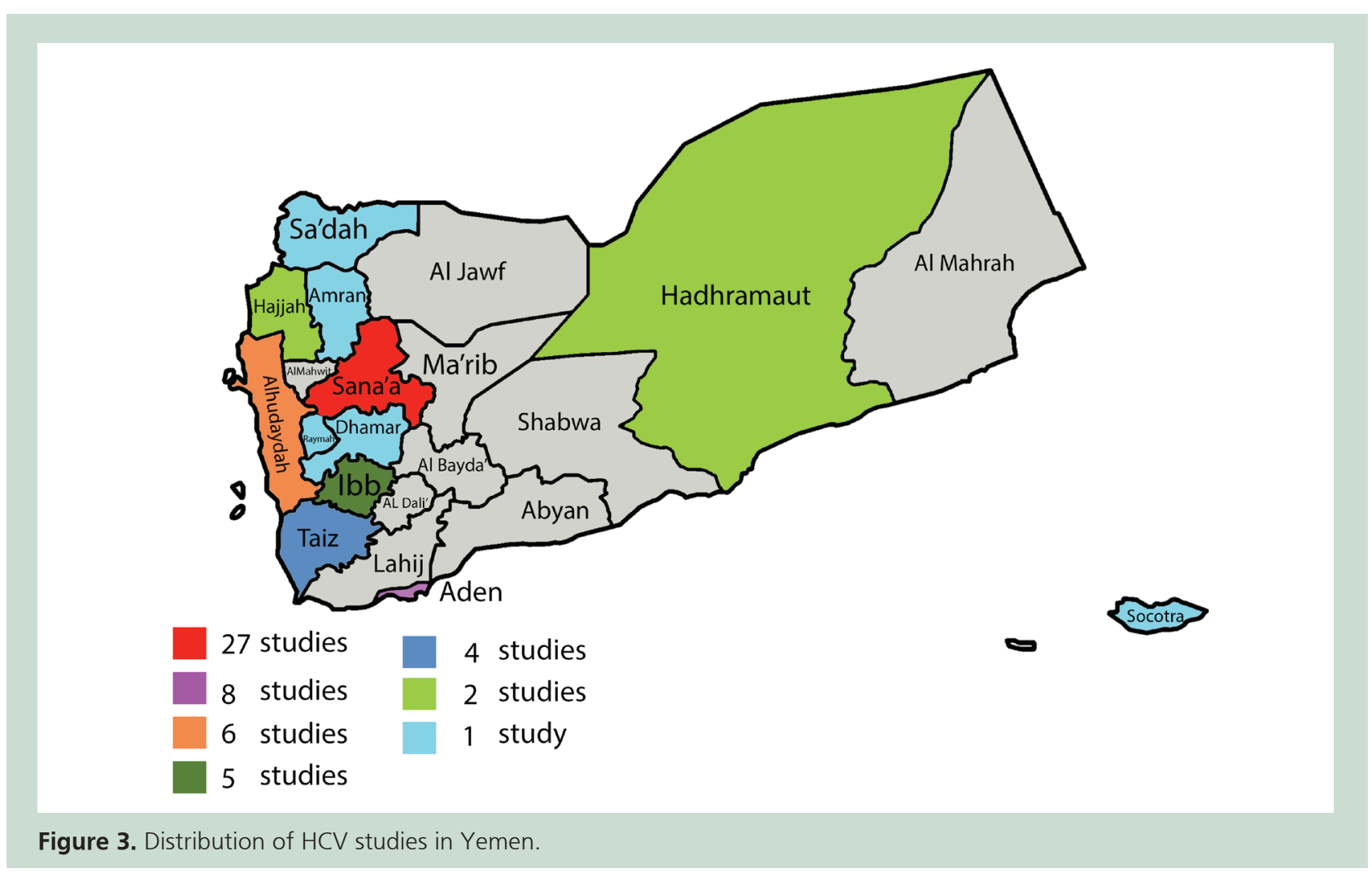


Table 1. General population group

\begin{tabular}{|c|c|c|c|c|c|c|c|c|c|c|}
\hline $\begin{array}{l}\text { Authors, } \\
\text { References }\end{array}$ & $\begin{array}{l}\text { Year of } \\
\text { Sample } \\
\text { Collection }\end{array}$ & $\begin{array}{l}\text { Year of } \\
\text { Publishing }\end{array}$ & $\begin{array}{l}\text { Type of } \\
\text { Study }\end{array}$ & $\begin{array}{l}\text { Sample } \\
\text { Size }\end{array}$ & $\begin{array}{l}\text { Prevalence of } \\
\mathrm{HCV}\end{array}$ & $\begin{array}{l}\text { Prevalence } \\
\text { by } \\
\text { Gender }\end{array}$ & $\begin{array}{l}\text { Study } \\
\text { Site }\end{array}$ & $\begin{array}{l}\text { Governo- } \\
\text { rate }\end{array}$ & Population & $\begin{array}{l}\text { Test } \\
\text { Type }\end{array}$ \\
\hline $\begin{array}{l}\text { Scott et al. } \\
\text { (12) }\end{array}$ & 1988 & 1992 & CS & 348 & $6 \%$ & $\begin{array}{l}\mathrm{M}=2.8 \%, \\
\mathrm{~F}=2.2\end{array}$ & $\begin{array}{l}\text { Schools, } \\
\text { houses }\end{array}$ & $\begin{array}{l}\text { Sana'a, } \\
\text { Hajjah, } \\
\text { Taiz, } \\
\text { Alhudaydah }\end{array}$ & Public & $\begin{array}{l}\text { ELISA, } \\
\text { RIBA* }\end{array}$ \\
\hline $\begin{array}{l}\text { El Guneid. } \\
\text { et al. (13) }\end{array}$ & $\mathrm{Na}$ & 1993 & CS & 294 & $1.00 \%$ & $\mathrm{Na}$ & $\begin{array}{l}\text { Blood } \\
\text { bank }\end{array}$ & Taiz & $\begin{array}{l}\text { Blood } \\
\text { doners } \\
\text { (as control) }\end{array}$ & EIA \\
\hline $\begin{array}{l}\text { El Guneid } \\
\text { et al. (13) }\end{array}$ & $\mathrm{Na}$ & 1993 & CS & 243 & $3.30 \%$ & $\mathrm{Na}$ & Hospital & Taiz & $\begin{array}{l}\text { Pregnant } \\
\text { women } \\
\text { (as control) }\end{array}$ & EIA \\
\hline $\begin{array}{l}\text { Gray et al. } \\
\text { (14) }\end{array}$ & 1992 & 1999 & CS & 627 & $0.30 \%$ & $\mathrm{Na}$ & Houses & $\begin{array}{l}\text { lbb, } \\
\text { Hajjah }\end{array}$ & Public & EIA \\
\hline $\begin{array}{l}\text { Al-Moslih \& } \\
\text { Al-Huraibi } \\
\text { (15). }\end{array}$ & $\mathrm{Na}$ & 2001 & CS & 120 & $4.20 \%$ & $\mathrm{Na}$ & Hospital & Sana'a & $\begin{array}{l}\text { Public } \\
\text { (health } \\
\text { control) }\end{array}$ & ELISA \\
\hline $\begin{array}{l}\text { Sallam et al. } \\
\text { (16) }\end{array}$ & $\mathrm{Na}$ & 2003 & CS & $\begin{array}{l}\text { Aden }=494 \\
\text { Sana' } a=493\end{array}$ & $\begin{array}{l}\text { Aden }=0.6, \\
\text { Sana' } a=0.2\end{array}$ & $\mathrm{Na}$ & $\begin{array}{l}\text { Blood } \\
\text { bank }\end{array}$ & $\begin{array}{l}\text { Sana'a, } \\
\text { Aden }\end{array}$ & $\begin{array}{l}\text { Blood } \\
\text { donors }\end{array}$ & $\begin{array}{l}\text { EIA, } \\
\text { RIBA* }^{*}\end{array}$ \\
\hline $\begin{array}{l}\text { Sallam et al. } \\
\text { (16) }\end{array}$ & $\mathrm{Na}$ & 2003 & CS & $\begin{array}{l}\text { African Ethnic=97, } \\
\text { and } \\
\text { Soqotra Island=99 }\end{array}$ & $\begin{array}{l}\text { African }=5.2 \%, \\
\text { Sogotra Island }=5.1 \%\end{array}$ & $\begin{array}{l}\text { African } \\
\text { Residents } \\
(\mathrm{F}=4.3 \%, \\
\mathrm{M}=5.9 \%), \\
\text { Soqotra, } \\
(\mathrm{F}=2 \%, \\
\mathrm{M}=7.8 \%)\end{array}$ & $\begin{array}{l}\text { Blood } \\
\text { bank }\end{array}$ & $\begin{array}{l}\text { Sana'a, } \\
\text { Socotra } \\
\text { İsland }\end{array}$ & Public & $\begin{array}{l}\text { EIA, } \\
\text { RIBA* }^{*}\end{array}$ \\
\hline $\begin{array}{l}\text { Sultan } \\
\text { et al. (17) }\end{array}$ & 2008-2010 & 2010 & CS & 3602 & $1.05 \%$ & $M=1.05 \%$ & $\begin{array}{l}\text { Blood } \\
\text { bank }\end{array}$ & Sana'a & $\begin{array}{l}\text { Blood } \\
\text { donors }\end{array}$ & MEIA \\
\hline $\begin{array}{l}\text { Bin Selm } \\
\text { (18) }\end{array}$ & 2007 & 2010 & CS & 100 & $0.80 \%$ & $\mathrm{Na}$ & $\begin{array}{l}\text { Blood } \\
\text { bank }\end{array}$ & Aden & $\begin{array}{l}\text { Blood } \\
\text { donors } \\
\text { (as control) }\end{array}$ & NA \\
\hline $\begin{array}{l}\text { Al-Shamiri } \\
\text { et al. (19) }\end{array}$ & 2007-2009 & 2011 & CS & 214 & $2.80 \%$ & $\mathrm{Na}$ & Schools & Taiz & Children & NA \\
\hline $\begin{array}{l}\text { Saghir et al. } \\
(20)\end{array}$ & 2008- 2010 & 2012 & CS & 1483 & $0.79 \%$ & $\mathrm{Na}$ & $\begin{array}{l}\text { Blood } \\
\text { bank }\end{array}$ & Alhudaydah & $\begin{array}{l}\text { Blood } \\
\text { donors }\end{array}$ & ICT \\
\hline Alodini (21) & 2010 & 2012 & CS & 3000 & $3 \%$ & $\mathrm{Na}$ & $\begin{array}{l}\text { Blood } \\
\text { bank }\end{array}$ & Sana'a & $\begin{array}{l}\text { Blood } \\
\text { donors }\end{array}$ & ELISA \\
\hline $\begin{array}{l}\text { Al-Waleedi \& } \\
\text { Khader (22) }\end{array}$ & 2007 & 2012 & CS & 469 & $1.30 \%$ & $\mathrm{Na}$ & $\begin{array}{l}\text { Blood } \\
\text { bank }\end{array}$ & Aden & $\begin{array}{l}\text { Blood } \\
\text { donors }\end{array}$ & EIA \\
\hline $\begin{array}{l}\text { Gacche \& } \\
\text { Al Mohani } \\
\text { (23) }\end{array}$ & 2010 & 2012 & CS & 554 & $1.99 \%$ & $\begin{array}{l}M=0.18 \%, \\
F=1.81 \%\end{array}$ & Hosues & $\mathrm{ibb}$ & Public & $\begin{array}{l}\text { ICT, } \\
\text { EIA* }\end{array}$ \\
\hline $\begin{array}{l}\text { Gacche \& } \\
\text { Al-Mohani } \\
\text { (24) }\end{array}$ & 2010-2011 & 2012 & CS & 2379 & $1.30 \%$ & $\begin{array}{l}M=0.29 \%, \\
F=1.01 \%\end{array}$ & Houses & $\mathrm{lbb}$ & Public & $\begin{array}{l}\text { ICT, } \\
\text { ELISA* }\end{array}$ \\
\hline $\begin{array}{l}\text { Murad } \\
\text { et al. (25) }\end{array}$ & 2011 & 2013 & CS & 400 & $8.50 \%$ & $\mathrm{Na}$ & Hospital & Sana'a & $\begin{array}{l}\text { Pregnant } \\
\text { women }\end{array}$ & ELISA \\
\hline $\begin{array}{l}\text { Al-Nabehi } \\
\text { et al. } \\
\text { (26) }\end{array}$ & $\mathrm{Na}$ & 2015 & CS & 501 & $\begin{array}{l}\text { Sana'a (1.5\%), } \\
\text { Taiz (0.6\%) } \\
\text { and Aden (0\%). }\end{array}$ & $\mathrm{Na}$ & $\begin{array}{l}\text { High, } \\
\text { schools } \\
\text { universities }\end{array}$ & $\begin{array}{l}\text { Sana'a, } \\
\text { Aden, } \\
\text { Taiz }\end{array}$ & Public & ELISA \\
\hline $\begin{array}{l}\text { Al-Zubiery } \\
\text { et al. (27) }\end{array}$ & 2016 & 2017 & CS & 11374 & $1 \%$ & $\begin{array}{l}M=1 \%, \\
F=0.9 \%\end{array}$ & $\begin{array}{l}\text { Blood } \\
\text { bank }\end{array}$ & Sana'a & $\begin{array}{l}\text { Blood } \\
\text { donors }\end{array}$ & ECLIA \\
\hline $\begin{array}{l}\text { Al-Zubiery } \\
\text { et al. (28) }\end{array}$ & 2016-2017 & 2017 & CS & 3606 & $1.60 \%$ & $M=1.60 \%$ & $\begin{array}{l}\text { Blood } \\
\text { bank }\end{array}$ & Sana'a & $\begin{array}{l}\text { Blood } \\
\text { donors }\end{array}$ & EIA \\
\hline $\begin{array}{l}\text { Al-Kadassy } \\
\text { et al. (29) }\end{array}$ & 2012-2015 & 2017 & CS & 1286 & $1.60 \%$ & $M=1.6 \%$ & Houses & Alhudaydah & Public & ELISA \\
\hline $\begin{array}{l}\text { Al-Hatheq } \\
\text { et al. (30) }\end{array}$ & 2018 & 2019 & CS & 500 & $2.80 \%$ & $\mathrm{Na}$ & $\begin{array}{l}\text { Blood } \\
\text { bank }\end{array}$ & Amran & $\begin{array}{l}\text { Blood } \\
\text { donors }\end{array}$ & $\begin{array}{l}\text { ICT, } \\
\text { ELISA* }\end{array}$ \\
\hline $\begin{array}{l}\text { Ghaleb } \\
\text { et al. (31) }\end{array}$ & 2017 & 2020 & CS & 340 & $1.20 \%$ & $\mathrm{Na}$ & $\begin{array}{l}\text { Blood } \\
\text { bank }\end{array}$ & Sana'a & $\begin{array}{l}\text { Blood } \\
\text { doners }\end{array}$ & $\begin{array}{l}\text { ECLIA, } \\
\text { ELISA* }\end{array}$ \\
\hline $\begin{array}{l}\text { Bin Barkat \& } \\
\text { Naseeb (32) }\end{array}$ & 2019 & 2020 & CS & 300 & $0.07 \%$ & $\mathrm{~F}=0.07 \%$ & Clinics & Hadhramaut & $\begin{array}{l}\text { Pregnant } \\
\text { women }\end{array}$ & ELISA \\
\hline
\end{tabular}

* $=$ Confirmed test

HCV: Hepatitis C virüs, ELISA: Enzyme-linked immunosorbent assay, RIBA: Recombinant immunoblot assay, ElA: Enzyme immunoassay, MEIA: Microparticle enzyme immunoassay, NA: Not available, ICT: Immune-chromatographic technique, ECLIA: Electrochemiluminescence immunoassay analyzer, CS: Cross-sectional, F: Female, M: Male, 
ies ranged from 30 to 11374 participate. On the other hand, seven different techniques used to test HCV in the 46 studies where 3 studies did not clarify what technic was used. Twenty-nine studies used Enzyme-Linked Immunosorbent Assay (ELISA) as the main test for HCV and 3 studies used it as a confirmed test. Enzyme immunoassay (EIA) was used in 8 studies as main and in one study was as a confirmed test. The immune-chromatographic technique (ICT) in five studies, electrochemiluminescence immunoassay analyzer (ECLIA) in two studies, but each of microparticle enzyme immunoassay (MEIA), and Chemiluminescent Microparticle Immunoassay (CMIA) was used only one study. While recombinant Immunoblot Assay (RIBA) and Polymerase Chain Reaction (PCR) used only as a confirmed test. RIBA was in 3 studies and PCR was in one study. Besides that, all the articles were HCV prevalence only there were not any incidence studies.

The prevalence of HCV was different according to the population groups as we divided the population into four groups, the prevalence according population is:

\section{General Group (low risk group)}

In the general group of the population, the prevalence was ranged between $0.07 \%-8.5 \%$. about twenty-one studies were done on the general population as the main group or control group. wherein 11 studies blood donors were the sample population two of them were control groups and the range of HCV prevalence in blood donors was $(0.2 \%-3.0 \%)$ with the mean of $1.27 \%$. Also, eight studies on the public one of them were the control group and the range of $\mathrm{HCV}$ prevalence was $(0 \%-6 \%)$ with the mean $2.8 \%$. For the pregnant women, only three studies were done on them one of them as a control group and the prevalence range was $0.07 \%-8.5 \%$ with the mean of the HCV prevalence $3.95 \%$. On the other hand, just one study was done on the children with a prevalence of 2.8\% (Table 1).

\section{Intermediate Risk Group}

In the intermediate-risk group, three studies were done on the health workers and dental clinic, and public health center cleaners. Where HCV prevalence was ranged between 1.03\%-3.5\% (Table 2).

\section{High Risk Group}

In the high-risk group, about 17 studies were done with a range between $6.4 \%-62.7 \%$. All these studies are patients but with different diseases. For the hemodialysis patients, 8 studies show us the range between $10.7 \%$ $27.6 \%$ and the mean $19.8 \%$. And for Hepatocellular carcinoma five studies are found with range $28.4 \%-43.7 \%$ and mean $37.5 \%$. On the other hand, 4 studies had liver disease patients as sample group one is acute liver disease and one chronic liver disease with prevalence ranged between $6.4 \%-62.7 \%$ with the mean $33.3 \%$ (Table 3).

\section{Patients Group}

Each of the other patients' groups has one study except non-Hodgkin's lymphomas patients which are two studies. The other patients are chronic renal failure, renal failure, non-Hodgkin's lymphomas, leprous, cataract surgery, diabetes, endoscopic, eye camps, sickle cell anemia, surgical department, and clinic visitors. The range of HCV prevalence was between $0 \%-46 \%$.

\section{DISCUSSION}

In our systematic review, it was noted in the last decade that there has been an increase in research activity in hepatitis $C$, where the number of researches was nearly three times that in the first two decades, and this indicates a high awareness of the seriousness hepatitis $C$, but most of this research was limited to the city of Sana'a for being the capital of Yemen and a suitable city for researchers to work where it provides a better level of

\begin{tabular}{|c|c|c|c|c|c|c|c|c|c|c|}
\hline $\begin{array}{l}\text { Authors, } \\
\text { References }\end{array}$ & $\begin{array}{l}\text { Year of } \\
\text { Sample } \\
\text { Collection }\end{array}$ & $\begin{array}{l}\text { Year of } \\
\text { Publishing }\end{array}$ & $\begin{array}{l}\text { Type of } \\
\text { Study }\end{array}$ & $\begin{array}{l}\text { Sample } \\
\text { Size }\end{array}$ & $\begin{array}{l}\text { Prevalence of } \\
\mathrm{HCV}\end{array}$ & $\begin{array}{l}\text { Prevalence } \\
\text { by } \\
\text { Gender }\end{array}$ & $\begin{array}{l}\text { Study } \\
\text { Site }\end{array}$ & $\begin{array}{l}\text { Governo- } \\
\text { rate }\end{array}$ & $\begin{array}{l}\text { Population } \\
\text { (Workers of) }\end{array}$ & $\begin{array}{l}\text { Test } \\
\text { Type }\end{array}$ \\
\hline $\begin{array}{l}\text { Shidrawi et al. } \\
\text { (33) }\end{array}$ & NA & 2004 & $\mathrm{CS}$ & 567 & $3.50 \%$ & NA & Hospital & Sana'a & Healthcare & EIA \\
\hline $\begin{array}{l}\text { Al-Kebsi et al. } \\
\text { (34) }\end{array}$ & 2014 & 2017 & CS & 509 & $1.60 \%$ & $\begin{array}{l}M=1.2 \% \\
F=1.9 \%\end{array}$ & Dental clinics & Sana'a & Dental clinic & ELISA \\
\hline $\begin{array}{l}\text { Al-Marrani \& } \\
\text { Al-Shamahy (35) }\end{array}$ & 2015 & 2018 & CS & 388 & $1.03 \%$ & $\begin{array}{l}M=0.6 \% \\
F=2.9 \%\end{array}$ & $\begin{array}{l}\text { Hospital, health } \\
\text { centers }\end{array}$ & Sana'a & $\begin{array}{l}\text { Public health } \\
\text { center cleaners }\end{array}$ & ELISA \\
\hline
\end{tabular}

${ }^{*}=$ Confirmed test.

HCV: Hepatitis C virus, ELISA: Enzyme-linked immunosorbent assay, EIA: Enzyme immunoassay, CS: Cross-sectional, F: Female, M: Male, 
services than other cities and most of the patients come to it to receive treatment there. On the other hand, we cannot rely on this epidemiological information to give us a correct idea of the prevalence of the hepatitis $C$ virus because most of this research focused on only 12 cities and neglected the rest of the Yemeni governorates. Also, the difference in techniques used in hepatitis $C$ tests may be an influencing factor on the true prevalence of hepatitis $C$, as there was a difference in the use of techniques in the research conducted on the prevalence rate (60). It was also noted that there were no researches on the incidence of $\mathrm{HCV}$, while all researches were on the prevalence of hepatitis $C$ virus. also, There was not any difference in the HCV prevalence between the gender in Yemen.
The prevalence of HCV was different according to the population group. According to the WHO report 2017 the prevalence of $\mathrm{HCV}$ infection in $\mathrm{WHO}$ regions vary from $0.5 \%$ to $2.3 \%$ which indicates that the prevalence in Yemen is high and the Yemeni population is considered at risk of HCV infection. In the general population, the prevalence was less than in Central Asia but it is more than that in the middle east and north Africa MENA $(61,62)$. In the healthy blood donors group, the prevalence in Yemen was close to that in Iraq, but compared to those in gulf countries (Kuwait, Qatar, Saudi Arabia ) it was considered to be lower except that in Oman (63). Also, the prevalence of $\mathrm{HCV}$ in blood donors in Yemen consider higher than that in Somalia (64). Unfortunately, the prevalence of $\mathrm{HCV}$ in pregnant women was higher

\section{Table 3. High risk group population}

\begin{tabular}{|c|c|c|c|c|c|c|c|c|c|c|}
\hline $\begin{array}{l}\text { Authors, } \\
\text { References }\end{array}$ & $\begin{array}{l}\text { Year of } \\
\text { Sample } \\
\text { Collection }\end{array}$ & $\begin{array}{l}\text { Year of } \\
\text { Publishing }\end{array}$ & $\begin{array}{l}\text { Type of } \\
\text { Study }\end{array}$ & $\begin{array}{l}\text { Sample } \\
\text { Size }\end{array}$ & $\begin{array}{l}\text { Prevalence of } \\
\mathrm{HCV}\end{array}$ & $\begin{array}{l}\text { Prevalence } \\
\text { by } \\
\text { Gender }\end{array}$ & $\begin{array}{l}\text { Study } \\
\text { Site }\end{array}$ & $\begin{array}{l}\text { Governo- } \\
\text { rate }\end{array}$ & $\begin{array}{l}\text { Population } \\
\text { (Patients } \\
\text { of) }\end{array}$ & $\begin{array}{l}\text { Test } \\
\text { Type }\end{array}$ \\
\hline $\begin{array}{l}\text { El Guneid } \\
\text { et al. (13) }\end{array}$ & NA & 1993 & CS & 108 & $21.50 \%$ & NA & NA & NA & $\begin{array}{l}\text { Acute liver } \\
\text { hepatitis }\end{array}$ & EIA \\
\hline $\begin{array}{l}\text { Gunaid et al. } \\
\text { (36) }\end{array}$ & NA & 1997 & CS & 78 & $6.40 \%$ & NA & Hospital & Aden & $\begin{array}{l}\text { Chronic liver } \\
\text { disease }\end{array}$ & ELISA \\
\hline $\begin{array}{l}\text { Al-Moslih \& } \\
\text { Al-Huraibi (15) }\end{array}$ & NA & 2001 & CS & 143 & $37.10 \%$ & NA & Hospital & Aden & Hemodialysis & NA \\
\hline $\begin{array}{l}\text { Al-Selwi et al. } \\
\text { (37) }\end{array}$ & $2004-2007$ & 2009 & CS & 54 & $27.60 \%$ & NA & Hospital & $\mathrm{lbb}$ & Hemodialysis & ELISA \\
\hline Bin Selm (38) & 2007 & 2010 & CS & 76 & $17.90 \%$ & NA & Hospital & Sana'a & Hemodialysis & ELISA \\
\hline Bin Selm (18) & 2007 & 2010 & CS & 51 & $62.70 \%$ & $\begin{array}{l}M=39.2 \% \\
F=23.5 \%\end{array}$ & Hospital & $\begin{array}{l}\text { Alhudaydah, } \\
\text { Raymah, } \\
\text { Sa'dah }\end{array}$ & Hemodialysis & ELISA \\
\hline Bin Selm (39) & 2006-2007 & 2011 & CS & 316 & $43.70 \%$ & $\begin{array}{l}M=62 \% \\
F=12 \%\end{array}$ & Hospital & Aden & Hemodialysis & NA \\
\hline $\begin{array}{l}\text { Saeed et al. } \\
(40)\end{array}$ & $2008-2010$ & 2012 & CS & 88 & $28.40 \%$ & $\begin{array}{l}M=28.1 \%, \\
F=28.6 \%\end{array}$ & Hospital & Sana'a & Hemodialysis & $\begin{array}{l}\text { ELISA, } \\
\text { PCR* }\end{array}$ \\
\hline $\begin{array}{l}\text { Salem et al. } \\
\text { (41) }\end{array}$ & 2001- 2008 & 2012 & CS & 251 & $38.20 \%$ & NA & Hospital & Dhamar & Hemodialysis & ELISA \\
\hline Baghza (42) & 2010 & 2014 & CS & 200 & $22.50 \%$ & $\begin{array}{l}M=60 \% \\
F=40 \%\end{array}$ & Hospital & Sana'a & Hemodialysis & ELISA \\
\hline $\begin{array}{l}\text { Aman et al. } \\
(43)\end{array}$ & $2000-2013$ & 2015 & CS & 219 & $40.20 \%$ & NA & Hospital & Sana'a & $\begin{array}{l}\text { Hepatocellular } \\
\text { carcinoma }\end{array}$ & ELISA \\
\hline $\begin{array}{l}\text { Al-Haimi et al. } \\
\text { (44) }\end{array}$ & 2009-2013 & 2016 & CS & 120 & $54.20 \%$ & NA & Hospital & Sana'a & $\begin{array}{l}\text { Hepatocellular } \\
\text { carcinoma }\end{array}$ & ELISA \\
\hline $\begin{array}{l}\text { Gaballah et al. } \\
\text { (45) }\end{array}$ & NA & 2018 & CS & 30 & $16.70 \%$ & $\begin{array}{l}M=63.3 \% \\
F=36.6 \%\end{array}$ & Hospitals & $\begin{array}{l}\text { Sana'a, } \\
\text { Aden, } \\
\text { Hadhramaut }\end{array}$ & $\begin{array}{l}\text { Hepatocellular } \\
\text { carcinoma }\end{array}$ & ELISA \\
\hline $\begin{array}{l}\text { Amran et al. } \\
\text { (11) }\end{array}$ & 2018 & 2019 & $\mathrm{CC}$ & 202 & $9.90 \%$ & $\begin{array}{l}M=11.5 \%, \\
F=15 \%\end{array}$ & Hospital & Sana'a & $\begin{array}{l}\text { Hepatocellular } \\
\text { carcinoma }\end{array}$ & ELISA \\
\hline $\begin{array}{l}\text { Hanash et al. } \\
\text { (46) }\end{array}$ & 2016 & 2019 & $\mathrm{CS}$ & 159 & $10.70 \%$ & $\begin{array}{l}M=14.3 \% \\
F=8.7 \%\end{array}$ & Hospital & Sana'a & $\begin{array}{l}\text { Hepatocellular } \\
\text { carcinoma }\end{array}$ & ELISA \\
\hline Al-Yousofi (47) & 2018 & 2019 & CS & 349 & $17.20 \%$ & $\begin{array}{l}M=9.5 \% \\
F=7.7 \%\end{array}$ & Hospital & Sana'a & Liver disease & ELISA \\
\hline $\begin{array}{l}\text { Almezgagi } \\
\text { et al. (48) }\end{array}$ & 2013-2016 & 2020 & CS & 100 & $21 \%$ & $\begin{array}{l}M=14.28 \%, \\
F=29.54 \%\end{array}$ & Hospital & Taiz & Liver disease & EIA \\
\hline
\end{tabular}




\begin{tabular}{|c|c|c|c|c|c|c|c|c|c|c|}
\hline $\begin{array}{l}\text { Authors, } \\
\text { References }\end{array}$ & $\begin{array}{l}\text { Year of } \\
\text { Sample } \\
\text { Collection }\end{array}$ & $\begin{array}{l}\text { Year of } \\
\text { Publishing }\end{array}$ & $\begin{array}{l}\text { Type of } \\
\text { Study }\end{array}$ & $\begin{array}{l}\text { Sample } \\
\text { Size }\end{array}$ & $\begin{array}{l}\text { Prevalence of } \\
\mathrm{HCV}\end{array}$ & $\begin{array}{l}\text { Prevalence } \\
\text { by } \\
\text { Gender }\end{array}$ & $\begin{array}{l}\text { Study } \\
\text { Site }\end{array}$ & $\begin{array}{l}\text { Governo- } \\
\text { rate }\end{array}$ & $\begin{array}{l}\text { Population } \\
\text { (Patients } \\
\text { of) }\end{array}$ & $\begin{array}{l}\text { Test } \\
\text { Type }\end{array}$ \\
\hline Denis et al. (49) & 1988-1990 & 1994 & CS & $\begin{array}{l}1309 \\
\text { (From 7 } \\
\text { countries) }\end{array}$ & $\begin{array}{l}7.1 \% \\
(\text { Yemenis = 21\%) }\end{array}$ & $\begin{array}{l}(F=8.9 \% \\
M=5.5 \%)\end{array}$ & NA & NA & Leprous & $\begin{array}{l}\text { EIA, } \\
\text { RIBA* }\end{array}$ \\
\hline Salem (50) & 2005-2007 & 2009 & CS & 192 & $15.10 \%$ & $\begin{array}{l}M=17.6 \% \\
F=10.7 \%\end{array}$ & Hospital & Sana'a & $\begin{array}{l}\text { Non-Hodgkin's } \\
\text { lymphoma }\end{array}$ & ELISA \\
\hline Salem (50) & 2005-2007 & 2009 & CS & 20329 & $4 \%$ & $\begin{array}{l}M=4.3 \%, \\
F=3.5 \%\end{array}$ & Hospital & Sana'a & $\begin{array}{l}\text { Other clinics } \\
\text { as control }\end{array}$ & ELISA \\
\hline $\begin{array}{l}\text { Al-Mansoob } \\
\text { et al. (51) }\end{array}$ & 2009-2011 & 2013 & CS & 394 & $14.20 \%$ & $\begin{array}{l}M=6.8 \%, \\
F=7.4 \%\end{array}$ & Hospital & Sana'a & $\begin{array}{l}\text { Surgical } \\
\text { department }\end{array}$ & ELISA \\
\hline $\begin{array}{l}\text { Thabet et al. } \\
\text { (52) }\end{array}$ & NA & 2014 & CS & 50 & $10 \%$ & $\begin{array}{l}M=60 \%, \\
F=40 \%\end{array}$ & Hospital & NA & Diabetes & ELISA \\
\hline $\begin{array}{l}\text { Al-Hegami et al. } \\
\text { (53) }\end{array}$ & 2013-2014 & 2015 & CS & 243 & $46.01 \%$ & $\begin{array}{l}M=40.13 \%, \\
F=62.5 \%\end{array}$ & Hospital & Aden & $\begin{array}{l}\text { Chronic renal } \\
\text { failure }\end{array}$ & ELISA \\
\hline $\begin{array}{l}\text { Al Awfi et al. } \\
\text { (54) }\end{array}$ & 2011-2015 & 2017 & CS & 278 & $1.07 \%$ & $\begin{array}{l}M=1.18 \%, \\
F=0.92 \%\end{array}$ & $\begin{array}{l}\text { Blood } \\
\text { dialysis } \\
\text { center }\end{array}$ & Alhudaydah & Renal failure & ELISA \\
\hline $\begin{array}{l}\text { Al-Kadassy } \\
\text { et al. (55) }\end{array}$ & 2014-2016 & 2018 & CS & 121 & $0 \%$ & $\begin{array}{l}M=0 \%, \\
F=0 \%\end{array}$ & Hospital & Alhudaydah & $\begin{array}{l}\text { Sickle cell } \\
\text { anemia }\end{array}$ & ELISA \\
\hline $\begin{array}{l}\text { Ghanem \& } \\
\text { Al-Rabeei (56) }\end{array}$ & 2017 & 2018 & CS & 1592 & $1.50 \%$ & $\begin{array}{l}M=12.8 \% \\
F=17.9 \%\end{array}$ & Patients & Sana'a & $\begin{array}{l}\text { Endoscopic } \\
\text { clinic }\end{array}$ & $\begin{array}{l}\text { ELISA, } \\
\text { PCR* }\end{array}$ \\
\hline Alhaj et al. (57) & 2014-2015 & 2018 & CS & 144 & $2.80 \%$ & $\begin{array}{l}M=3 \% \\
F=2.2 \%\end{array}$ & Hospital & Sana'a & $\begin{array}{l}\text { Non-Hodgkin's } \\
\text { lymphoma }\end{array}$ & CMIA \\
\hline $\begin{array}{l}\text { Al-Shaer et al. } \\
\text { (58) }\end{array}$ & 2016-2017 & 2019 & CS & 376 & $1.30 \%$ & NA & Patients & Sana'a, Ibb & Eye camps & ICT \\
\hline $\begin{array}{l}\text { Bamashmus \& } \\
\text { Abdulrahim (59) }\end{array}$ & 2012 & 2019 & CS & 1203 & $4.10 \%$ & $\begin{array}{l}M=44.9 \%, \\
F=55.1 \%\end{array}$ & $\begin{array}{l}\text { Eye } \\
\text { hospital }\end{array}$ & Sana'a & Cataract surgery & ELISA \\
\hline $\begin{array}{l}\text { Al-Hatheq et al. } \\
(30)\end{array}$ & 2018 & 2019 & CS & 300 & $28.00 \%$ & NA & $\begin{array}{l}\text { Blood } \\
\text { banks, } \\
\text { hospitals }\end{array}$ & Amran & Clinic visitors & $\begin{array}{l}\text { ICT, } \\
\text { ELISA* }\end{array}$ \\
\hline
\end{tabular}

$*=$ Confirmed test.

HCV: Hepatitis C virus, EIA: Enzyme immunoassay, RIBA: Recombinant immunoblot assay, ELISA: Enzyme-linked immunosorbent assay, CMIA: Chemiluminescent microparticle immunoassay, ICT: Immune-chromatographic technique, CS: Cross-sectional, F: Female, M: Male, NA: Not available.

than blood donors in Yemen and even that from the pregnant women in Saudi Arabia and Iraq, but compared with that in Egypt it considers lower $(63,65)$. Besides that, the prevalence of HCV in the Yemensi public is considered higher than that in Lebanon and Iraq (63). On the other hand, the children have only one study for the prevalence of $\mathrm{HCV}$ with $2.8 \%$ which is higher than that in Somalia, but lower than that in Egyptian children $(64,65)$. The situation in Yemen could be one of the reasons to have a high prevalence in the general population which they suppose to be the health group with a low prevalence.

In the intermediate-risk group in Yemen, the health workers have the highest prevalence of 3.5\%, then the dental worker $(1.6 \%)$, then the cleaners in health facilities $(1.03 \%)$ where the range of the prevalence in Yemen is close to that of Iraq, but in Iraq, the high prevalence of HCV was high in dental workers group (63). However, the range of HCV in Yemen was higher than in Jordon, and Kuwait, but lower than in Egypt $(63,65)$. This indicates that there is more need for adequate training of working safely for workers in the health facilities in Yemen and regularly test for HCV infection in this group as a control for safety.

In the high-risk group, the range of the prevalence of HCV in Yemen was up to $62.7 \%$ which is less than the range of the risk group of Fertile Crescent (63). Where the prevalence of hepatitis $C$ in the hemodialysis group considered higher than the prevalence in the Middle East and African regions (66-68). While it was less than Oman, Kuwait, and Saudi Arabia (69). But the prevalence of HCV in hemodialysis patients is close to that in Lebanon and Palestine (63). Also, it was found that the more number of hemodialysis times the more risk of getting infected with the virus in Yemenis patients (11). This high prevalence in hemodialysis considers the high-risk level of infections in the centers of hemodialysis in Yemenis patients where there are difficulties of controlling the in- 
fections because of the poor level of health. In chronic liver disease the prevalence was found only in one study where it is less than Somalia's and United Arab Emirates' prevalence, but in acute liver disease, it was higher than united Arab emirates and less than in Saudi Arabia $(64,69)$.

In the fourth group of patients, the diabetic patients had a prevalence more than that of Kuwait, but less than in Iraq and Egypt $(65,69)$. Beside in non-Hodgkin patient, the prevalence in Yemenis patients were lower than in Saudi Arabia and Egypt $(65,69)$. But for the hemolytic anemia, there was no relation with the infection of HCV where the prevalence was $0 \%$ but in the study in Jordon, it was $40.5 \%$ (63). Also, in the other patients, the prevalence of Yemenis patients was higher than Saudi Arabia and lower than Kuwaiti patients (69) we can figure that there is a need for doing tests for HCV for all patients to facilitate the elimination of HCV infections in Yemen.

In conclusion, we can say that there is an increase in the prevalence of hepatitis $C$ in Yemen in all population

\section{REFERENCES}

1. Axley $P$, Ahmed $Z$, Ravi S, Singal AK. Hepatitis C Virus and Hepatocellular Carcinoma: A Narrative Review. J Clin Transl Hepatol 2018;6:79-84.

2. Organization WH. Hepatitis C: fact sheet 27 July 2020. Available from: https://www.who.int/news-room/fact-sheets/detail/hepatitis-c\#: : :text=Key\%20facts, major\%20cause \%20of\%20liver\%20 cancer.

3. Stanaway JD, Flaxman AD, Naghavi M, et al. The global burden of viral hepatitis from 1990 to 2013: findings from the Global Burden of Disease Study 2013. Lancet 2016;388:1081-8.

4. Chemaitelly H, Mahmud S, Mumtaz GR, et al. Hepatitis C virus in the Middle East and North Africa: its epidemiology and the way forward In: Laher I, ed. Handbook of Healthcare in the Arab World. New York, NY: Springer International Publishing; 2019:1-27.

5. Organization WH. Global Hepatitis Report 2017: World Health Organization; 2017.

6. D'Ambrosio R, Degasperi E, Colombo M, Aghemo A. Direct-acting antivirals: the endgame for hepatitis C? Curr Opin Virol 2017;24:31-7.

7. Organization WH. Global health sector strategy on viral hepatitis 2016-2021. Towards ending viral hepatitis. World Health Organization, 2016.

8. Organization $\mathrm{WH}$. Combating hepatitis $\mathrm{B}$ and $\mathrm{C}$ to reach elimination by 2030: advocacy brief. World Health Organization, 2016.

9. Liberati A, Altman DG, Tetzlaff J, et al. The PRISMA statement for reporting systematic reviews and meta-analyses of studies that evaluate health care interventions: explanation and elaboration. J Clin Epidemiol 2009;62:e1-e34.

10. Houghton M. Discovery of the hepatitis C virus. Liver Int 2009;29:828.

11. Amran OAA, Al-Shamahy HA, Al Hadad AM, Jaadan BM. Explosion of hepatitis $B$ and $C$ viruses among hemadialysis patients as a result of hemodialysis crisis in Yemen. Universal Journal of Pharmaceutical Research 2019. DOI: 10.22270/ujpr.v4i5.311. groups compared to the global prevalence. The reasons for this are due to the current situation in Yemen, including war, and a decline in the level of living, economic, and health. Where there is an urgent need to support the health sector and make periodic checks for all patients who are at risk of getting infected with the virus due to blood transfusion or dialysis, and attention to providing treatment to patients also doing periodic checks for the workers in the health departments. There is also a need to expand the scope of research and pay attention to new research groups and other cities to get a more comprehensive idea of the prevalence of the hepatitis $C$ virus in Yemen. Knowing that awareness and raising awareness of prevention and hygiene methods and the importance of treatment for the disease will help Yemenis to reduce the prevalence and try to keep pace with the World Health Organization's plan to limit the hepatitis C virus infection in 2030.

Ethics statement: Our systematic review does not require any ethics approval as it relies entirely on previously published data.
12. Scott DA, Constantine NT, Callahan J, et al. The epidemiology of hepatitis $C$ virus antibody in Yemen. Am J Trop Med Hyg 1992;46:63-8.

13. el Guneid AM, Gunaid AA, O'Neill AM, et al. Prevalence of hepatitis $B, C$, and $D$ virus markers in Yemeni patients with chronic liver disease. J Med Virol 1993;40:330-3.

14. Gray GC, Kassira EN, Rodier GR, et al. Remote village survey for agents causing hepatosplenic disease in the Republic of Yemen. Trop Doct 1999;29:212-9.

15. Al-Moslih MI, Al-Huraibi MA. Prevalence of hepatitis C virus among patients with liver disease in the Republic of Yemen. East Mediterr Health J 2001;7(4-5):771-8.

16. Sallam TA, Tong CYW, Cuevas LE, et al. Prevalence of blood-borne viral hepatitis in different communities in Yemen. Epidemiol Infect 2003;131(1):771-5.

17. Sultan A, Abdulrahman A, Omar S, Huda S. Short communication the frequency of hepatitis $B$ and $C$ among blood donors: a hospital-based study in Sana'a, Yemen. Biohealth Science Bulletin 2010;2:65-68.

18. Selm SB. Prevalence of hepatitis $C$ virus infection among hemodialysis patients in a single center in Yemen. Saudi J Kidney Dis Transpl 2010;21(6):1165-8.

19. Al-Shamiri AH, Al-Taj MA, Ahmed AS. Prevalence and co-infections of schistosomiasis/hepatitis B and C viruses among school children in an endemic areas in Taiz, Yemen. Asian Pac J Trop Med 2011;4:404-8.

20. Saghir SAM, Al-Hassan FM, Alsalahi OSA, Alhariry A, Baqir HS. Frequencies of HBV, HCV, HIV, and syphilis markers among blood donors: A hospital-based study in Hodeidah, Yemen. Trop J Pharm Res 2012;11:132-6.

21. Alodini AQ. Prevalence of hepatitis $B$ virus (HBV) and hepatitis $C$ virus (HCV) infections among blood donors at Al-Thawra Hospital Sana'a City-Yemen. Yemeni J Med Sci 2014;6:16-20. 
22. Al-Waleedi AA, Khader YS. Prevalence of hepatitis B and C infections and associated factors among blood donors in Aden City, Yemen. East Mediterr Health J 2012;18:624-9.

23. Gacche RN, Kaid AM. Epidemiology of viral hepatitis B and C infections in ibb city, Yemen. Hepat Mon 2012;12:460-2.

24. Gacche RN, Al-Mohani SK. Seroprevalence and risk factors for hepatitis $C$ virus infection among general population in central region of Yemen. Hepat Res Treat 2012;2012:689726

25. Murad EA, Babiker SM, Gasim Gl, et al. Epidemiology of hepatitis $B$ and hepatitis $C$ virus infections in pregnant women in Sana'a, Yemen. BMC Pregnancy Childbirth 2013;13:127.

26. Al-Nabehi BAH, Al-Shamahy H, Saeed WSE, Musa AM, El Hassan AM, Khalil EAG. Sero-molecular epidemiology and risk factors of viral hepatitis in urban Yemen. International Journal of Virology 2015; 11:133-8.

27. Al-Zubiery T, Alharazi T, Alsumairy H, Al-Zubiery A. Sero-prevalence of Hbs Ag, HCV and HIV Among Blood Donors in Three Blood Bank Centers in Sana'a City: Yemen. Journal of Biotechnology and Biomedical Science 2017;1:46-52.

28. Al-Zubiery TK, Alharazi T, Alsumairy H, et al. Sero-prevalence of Anti-HCV among Yemenis blood donors attending national blood transfusion and research centre in Sana'a: Yemen. International Blood Research \& Reviews 2017;7:1-6.

29. Al-Kadassy AM, Balkam FA, Bashanfer S. Seroprevalence of Hepatitis B and C Infections among Adult Males in Beit al-Faqih District, Al-Hodeidah Governorate, Republic of Yemen. Journal of Advances in Medicine and Medical Research 2016;19:1-6.

30. Al-Hatheq A, Abakar AD, Al-Ofairi B. Seroprevalence of hepatitis C virus infection among blood donors and clinical visitors in Amran Governorate, Yemen. International Journal of Molecular Microbiology 2019;2:25-33.

31. Ghaleb YA, Alshahari AZ, Ali MA, et al. Prevalence and associated factors with transfusion-transmitted infections among blood donors at the national blood transfusion and research center, Sana'a, Yemen. 2020. DOI: 10.21203/rs.3.rs-116777/v1

32. Barkat B, Naseeb AA. Sero-prevalence and associated factors of viral hepatitis $B$ and $C$ infection among pregnant women in Alaeen Valley, Hadhramout Governorate, Yemen: Al-Razi University; 2020.

33. Shidrawi R, Al-Huraibi MA, Al-Haimi MA, et al. Seroprevalence of markers of viral hepatitis in Yemeni healthcare workers. J Med Virol 2004;73:562-5.

34. Al-Kebsi A, Othman A, Abbas A, et al. Sero-prevalence of hepatitis $C$ virus among dental clinic workers in Sana'a city-Yemen and the risk factors contributing for its infection. Univ J Pharm Res 2017;2:28-33

35. Al-Marrani WHM, Al-Shamahy HA. Prevalence of HBV and HCV; and their associated risk factors among public health center cleaners at selected Public Health Centers in Sana'a city-Yemen. Journal of Pharmaceutical Research 2018;3:58-62.

36. Gunaid AA, Nasher TM, el-Guneid AM, et al. Acute sporadic hepatitis in the Republic of Yemen. J Med Virol 1997;51:64-6.

37. Al-Selwi AHA, Elezzy Y, Al Ghazali J, Hadi S. Association of hepatocellular carcinoma with hepatic viral markers B and $C$ among Yemenis patients at Althawra Hospital Sana'a. Sudan Medical Journal. 2009;4:237-42.

38. Salem A. Bin Selm, Prevalence of hepatitis B and C viral markers in chronic liver disease patients: a single center experience from Yemen. Arab J Gastroenterol 2010;11:105-7.

39. Bin Selm SA. The seroprevalence of anti-HCV in high-risk dialysis patients. Saudi J Kidney Dis Transpl 2011;22:806-7.

40. Saeed NM, Bawazir AA, Al-Zuraiqi M, et al. Why is hepatocellular carcinoma less attributable to viral hepatitis in Yemen? Asian Pac J Cancer Prev 2012;13:3663-7.
41. Salem A, Abdulrab A, Alfakeh Y, Aown A. Hepatocellular carcinoma in Yemeni patients: a single centre experience over an 8-year period. East Mediterr Health J 2012;18:693-9.

42. Baghza NM. The prevalence of hepatitis $C$ virus among hemodialysis patients in Yemen. J Purity Utility React Environ 2014;3:62-6.

43. Aman K, Al-Dubai SA, Aman R, Hawash A, Alshagga M, Kassim $S$. Prevalence and associated factors of hepatitis $C$ virus infection among renal disease patients on maintenance hemodialysis in three health centers in Aden, Yemen: a cross sectional study. Saudi J Kidney Dis Transpl 2015;26:380-5.

44. Al-Haimi M, Hamid GA, Ahmed AS. Hepatocellular carcinoma in Yemen: Epidemiology and clinical presentation. World Journal of Pharmaceutical and Medical Research 2018;4:248-53.

45. Gaballah A, Shawky S, Elsawaf G, et al. Virological Profiles of HBV and HCV in Hepatocellular Carcinoma in Egypt and Yemen. Egyptian Journal of Medical Microbiology 2018;27:7-17.

46. Hanash SH, Al-Shamahy HA, Bamshmous MHS. Prevalence and genotyping of hepatitis $C$ virus in hemodialysis patients and evaluation of HCV-core antigen test in screening for dialysis in Sana'a city, Yemen. Universal J Pharm Res 2019;4:14-8.

47. Al-Yousofi MAQ. Prevalence of hepatitis $B$ and $C$ virüs among hemodialysis patient and infection control in dialysis units in Sana'a City, Yemen. Al-Razi University; 2019.

48. Almezgagi M, Edrees W, Al-Shehari W, et al. Prevalence of hepatitis $B$ virus and hepatitis $C$ virus and associated risk factors among hemodialysis patients in Ibb city-Yemen. PSM Microbiol 2020;5:3240 .

49. Denis F, Aussel L, Ranger S, et al. Prevalence of antibodies to hepatitis $C$ virus among patients with leprosy in several African countries and the Yemen. J Med Virol 1994;43:1-4.

50. Salem AK. Prevalence of HCV among Yemeni patients with non-Hodgkin's lymphoma at Al-Thawra teaching hospital. Gulf J Oncolog 2009(5):22-9.

51. Al-Mansoob AS, Salem AK, A-Selwi A, Assamawi A. Risk factors of hepatitis $B$ and $C$ viruses among patients admitted in surgical departments at Al-Thawra Hospital, Sana'a, Yemen. Sudan Medical Journal 2013;49:168-75.

52. Thabet HM, AL-Souraqy AM, Abbas AH, AL-hogary IQ, Anam EN, Noaman NS. The relationship of Hepatitis $C$ and $B$ with diabetes of Yemeni patients. Asian Pac J Health Sci. 2014;1(4):370-6.

53. Al-Hegami MA, Al-Mamari A, Al-Kadasse AS, et al. Prevalence and risk factors of hepatitis $B$ and hepatitis $C$ virus infections among patients with chronic renal failure in Zabeed city, Yemen Republic. Open Journal of Medical Microbiology 2015;5:136-42.

54. Al Awfi MS, Al-Kamarany MA, Mohsen R, et al. Hepatitis C Virus Infection among Chronic Renal Failure Patients Pre-Dialysis Therapy in Hodiedah City, Yemen. Microbiology Research Journal International. 2017:1-6.

55. Al-kadassy AM, Alkadasi MN, Puttauah E, Naji A, Alhwthyf A. Prevalence of hepatitis $B, C$ and HIV among sickle cell disease patients in Hodeidah city, YEMEN. J Bio Innov 2018;1:114-25.

56. Ghanem YA, Al-Rabeei NA. Prevalence of Hepatitis B and C Viral Infection among Patients Attending to the Endoscopic Clinic at Al-Thowrah Hospital in Sana'a City, Yemen. Global Journal of Gastroenterology \& Hepatology. 2018;6:1-4.

57. Nagi A. ALHaj SMA-A, Khaldoon S. Abdullah, Abdul Baki, Al-Robasi. Prevalence and risk factors of hepatitis $C$ virus infection among lymphoma patients in Sana'a city, Yemen. Global journal for Research Analysis 2018;7:31-3.

58. Al-Shaer MY, Bamashmus MA, Al-Akily SA, Al-Akhlee HA. Prevalence of hypertension, diabetes mellitus and Hepatitis B and $C$ among people seeking cataract surgery in eye camps in Yemen. Yemeni J Med Health Res. 2019;8:54-61. 
59. Bamashmus MA, Abdulrahim AM. Frequency of Hepatitis B, Hepatitis $C$ and Human Immunodeficiency virus in patients undergoing cataract surgery. Hadhramout University Journal of Natural \& Applied Sciences 2019;169-15.

60. Singh MN, Lal A, Poddar CK, Ejaz SK. Comparative evaluation of ELISA and rapid screening techniques for the diagnosis of HCV in haemodialysis patients in tertiary care hospital, South Bihar, India. Journal of Evolution of Medical and Dental Sciences 2017;6:66837.

61. Chaabna K, Cheema S, Abraham A, et al. Systematic overview of hepatitis $C$ infection in the Middle East and North Africa. World J Gastroenterol 2018;24:3038-54.

62. Botheju WSP, Zghyer F, Mahmud S, et al. The epidemiology of hepatitis $C$ virus in Central Asia: Systematic review, meta-analyses, and meta-regression analyses. Sci Rep 2019;9:1-15.

63. Chemaitelly H, Chaabna K, Abu-Raddad LJ. The epidemiology of hepatitis $C$ virus in the Fertile Crescent: systematic review and meta-analysis. PloS One 2015;10:e0135281.

64. Hassan-Kadle MA, Osman MS, Ogurtsov PP. Epidemiology of viral hepatitis in Somalia: Systematic review and meta-analysis study. World J Gastroenterol 2018;24:3927-57
65. Kouyoumjian SP, Chemaitelly H, Abu-Raddad LJ. Characterizing hepatitis $C$ virus epidemiology in Egypt: systematic reviews, meta-analyses, and meta-regressions. Sci Rep 2018;8:1661.

66. Harfouche M, Chemaitelly H, Mahmud S, et al. Epidemiology of hepatitis $C$ virus among hemodialysis patients in the Middle East and North Africa: systematic syntheses, meta-analyses, and meta-regressions. Epidemiol Infect 2017;145:3243-63.

67. Ashkani-Esfahani S, Alavian SM, Salehi-Marzijarani M. Prevalence of hepatitis $C$ virus infection among hemodialysis patients in the Middle-East: A systematic review and meta-analysis. World J Gastroenterol 2017;23:151-66.

68. Jadoul M, Bieber BA, Martin P, et al. Prevalence, incidence, and risk factors for hepatitis $C$ virus infection in hemodialysis patients. Kidney Int 2019;95:939-47.

69. Mohamoud YA, Riome S, Abu-Raddad LJ. Epidemiology of hepatitis $C$ virus in the Arabian Gulf countries: Systematic review and meta-analysis of prevalence. Int J Infect Dis 2016;46:116-25. 